\title{
Lymphoepithelioma-like carcinoma of the bladder: A case report
}

\author{
Konstantinos Stamatiou ${ }^{1}$, Georgios Christopoulos ${ }^{2}$, Aikaterini Tsavari ${ }^{3}$, Kallirhoe Koulia ${ }^{3}$, \\ Kassiani Manoloudaki ${ }^{3}$, Thivi Vassilakaki ${ }^{3}$ \\ ${ }^{1}$ Urology Department, Tzaneio Hospital Pireas, Greece; \\ ${ }^{2}$ Department of Internal Medicine, Tzaneio Hospital Pireas, Greece; \\ ${ }^{3}$ Pathology Department, Tzaneio Hospital Pireas, Greece.
}

\begin{abstract}
Summary Lymphoepithelioma is a rare histological type of low differentiated carcinoma of the nasopharyngeal region characterized by marked infiltration of lymphocytes in the area involved by tumor. However, carcinomas with this peculiar morphologic feature have been also described in various anatomic locations and they are generally designated «lymphoepithelioma-like carcinomas». Those of the urinary bladder are uncommon as they account of $0.4 \%-1.3 \%$ of all bladder carcinomas. They may coexist with the conventional urothelial carcinoma. Given their rarity, there is poor information regarding their behaviour. Here we present a new case of lymphoepithelioma-like carcinoma in the bladder and we discuss its characteristics and prognosis.
\end{abstract}

KEY WORDS: Lymphoepithelioma-like carcinoma; Bladder; Prognosis.

Submitted 24 April 2015; Accepted 21 May 2015

\section{INTRODUCTION}

Lymphoepithelioma also known as "class III nasopharyngeal carcinoma" is a rare form of undifferentiated carcinoma of the nasopharyngeal region that is considered a variant of undifferentiated non-keratinizing squamous cell carcinoma. It is characterized by excess inflammatory infiltrate of lymphocytes which appears to be intimately admixed with the carcinomatous cells. The last are large with vesicular nuclei and prominent nucleoli that are arranged in a dense growth pattern. This tumor displays a strong association with the Epstein Barr virus infection and its incidence exhibits a remarkable geographic variation $(1,2)$. Reason explaining the above peculiarity is practically unknown.

Lymphoepithelioma-like carcinomas (LELC) arise outside of the nasopharynx, but resemble a lymphoepithelioma both morphologically and histologically. They may be found in almost any organ containing epithelial tissue such as the lung, thymus, breast, colon, endometrium, prostate, and skin, as well as urinary bladder, trachea, esophagus, stomach, salivary glands, vulva (3). They may coexist with the conventional adenocarcinomas of the abovementioned organs and therefore they are classified according to the percentance of lymphoepithelioma component within the tumor. Therefore three types of LELC are described: pure $(100 \%)$, predominant $(>50 \%)$, or focal $(<50 \%)$ (3). Their occurrence in the urinary system is very rare and in fact, the reported incidence of LELC in the bladder is between $0.4 \%$ and $1.3 \%$ of all bladder carcinomas (4).

\section{Case presentation}

A 67-year-old woman suffering from recurrent urinary tract infections presented with macroscopic hematuria for 2 months. Urinalysis was non diagnostic for infection while urine cytology analysis showed markedly atypical urothelial cells. The patient underwent cystoscopic examination, which revealed a large tumor with mixed polypoid and solid- morphology, located in the right lateral wall, the trigon and the dome of the bladder. A computed tomography (CT) scan suggested locally advanced disease (Figure 1) however no lymph node involvement, non distant metastasis, and no hydronephrosis were shown. She refused surgical intervention; however, she subsequently agreed to receive treatment after an episode

\section{Figure 1.}

Computed tomography (CT) scan suggested locally advanced disease.

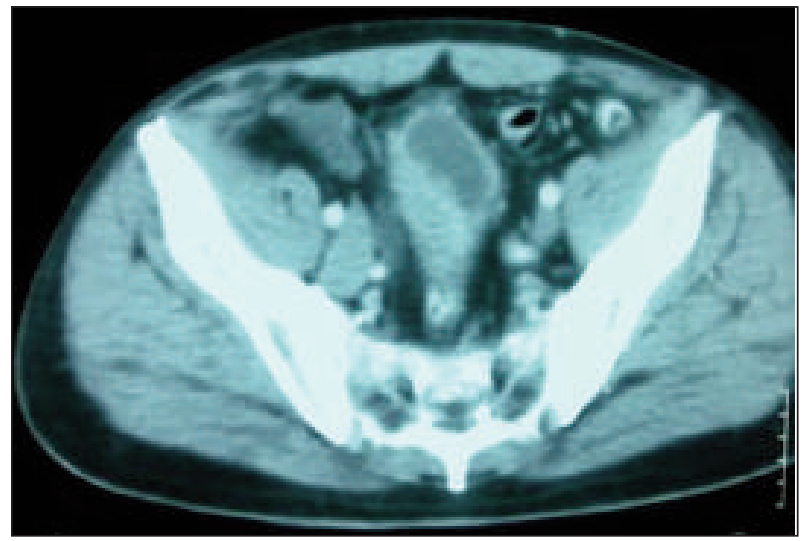

Archivio Italiano di Urologia e Andrologia 2016; 88, 2 
of recurrent gross hematuria accompanied by acute drop in hematocrit levels. The patient underwent an extensive transurethral resection of the bladder tumor (TUR-BT) in a single session. Sections of the TUR-BT showed invasive carcinoma, composed of predominant LELC $50 \%$ of the specimen) with grade 3 urothelial carcinoma (Figures 25). The patient was diagnosed with local invasive bladder tumor, and she was suggested to undergo cystectomy with ileal conduit formation. However due to special concerns regarding effects of surgery on her able-bodiedness she seeked organ-conserving approaches and she was referred to the oncological clinic. There she was advised that bladder-conserving approaches were not recommended because of the likely poor response to chemotherapy by the LELC elements, rendering thus radical cystectomy the standard of care. However, the woman continued to seek bladder preservation and presented to our institution to discuss bladder-sparing options. Due to the limited experience regarding treatment of this particular tumor, the patient was further

\section{Figure 2.}

Lymphoepithelioma-like carcinoma of the bladder infiltrating tumours cells and nests in an inflamatory background (H-Ex20).

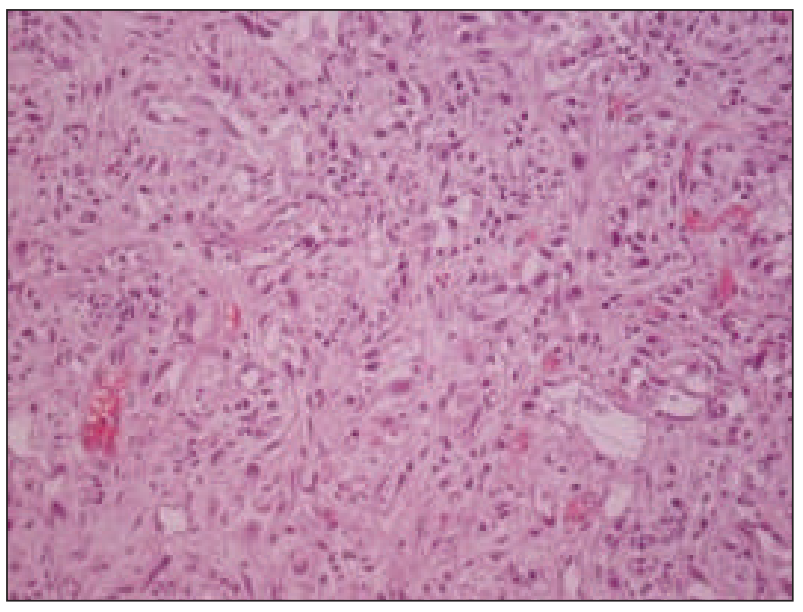

\section{Figure 3.}

The coexistent typical urothelial carcinoma in the same tumour (H-Ex20).

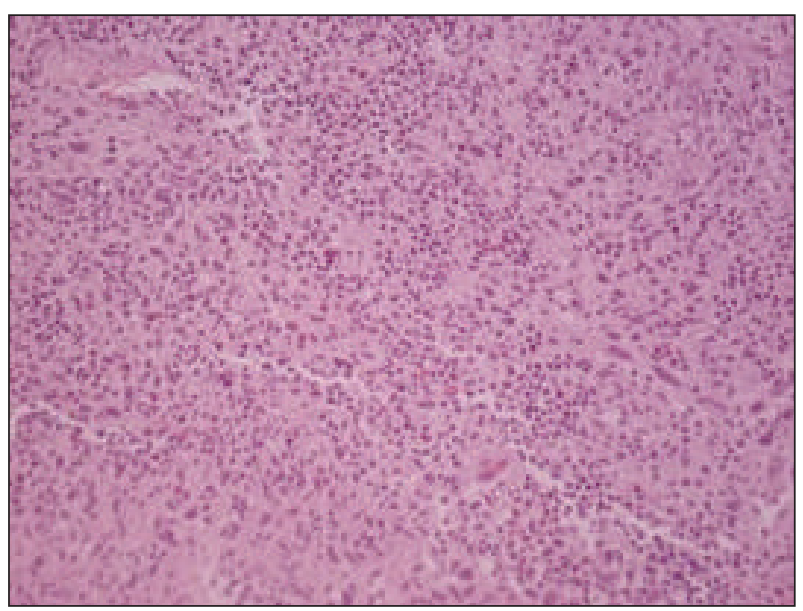

Figure 4.

Strong immunoreactivity against CKAE1-AE3 of tumours cells (x20).

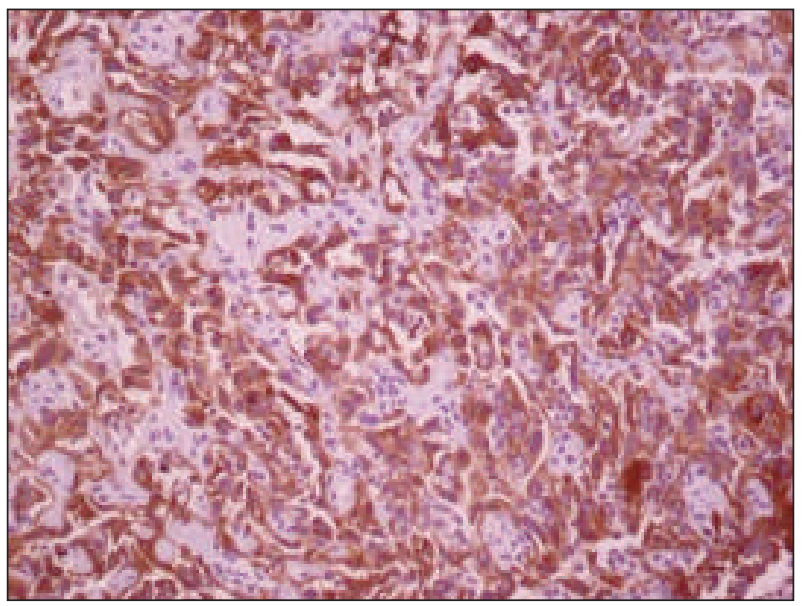

Figure 5.

CKAE1-AE3 in typical urothelial carcinoma. Comparative study (x20).

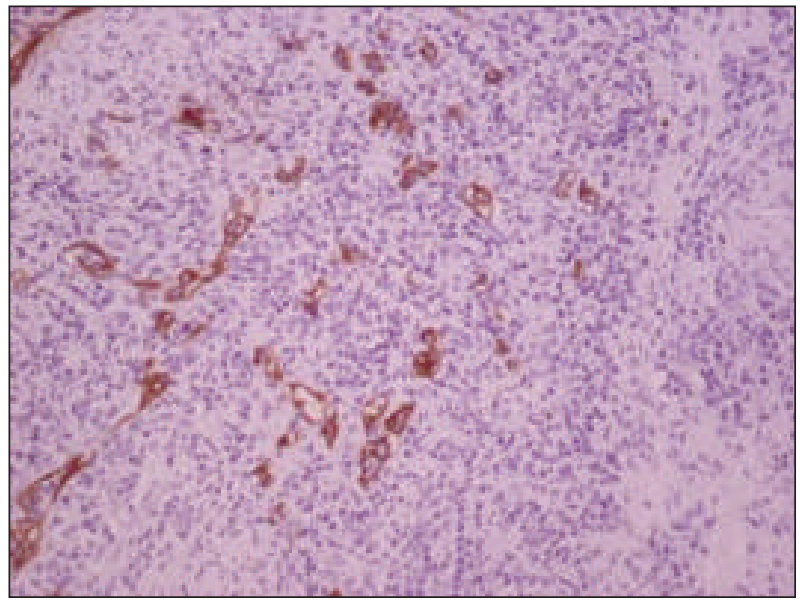

referred to the radiotherapy department. There she was advised to receive chemoradiation. Uncertainty regarding the outcome and additional consulting helped the patient to choose radical cystectomy. She did not receive adjuvant therapy. She is now under close observation with regular clinical and radiologic follow-up.

\section{Discussion}

Given their rarity, there is poor information regarding LECCs behaviour. However, urinary bladder carcinomas with a lymphoepithelioma-like component often manifest in T2-T3 stages and in 10\% to 15\% of the reported cases the cancer has spread to distant organs at the time of the first diagnosis. On the other hand, since these tumors constitute less than $1.5 \%$ of all carcinomas encountered at this anatomic location, no large systematically collected follow-up data exists and limited information regarding their biologic behaviour render treatment choice difficult (5). 
Therefore, the fact that most LECCs are discovered in advanced stages (T2-T3) could be rather attributed to the lack of specific symptoms than to a presuming aggressive behaviour.

In fact, evidence suggests that LELCs of the urinary tract may exhibit less malignant potential than the conventional urothelial carcinoma: it has been demonstrated that pure or predominant type responds better to chemotherapy than conventional urothelial carcinoma (6). In mixed cases of urinary bladder carcinomas and LELCs, the more the presence of lymphoepithelioma-like elements, the less the risk of death from the disease. More precisely, a 5-year survival rate of 59\% was reported for the predominant type while that of the pure type achieves $62 \%$ (7).

Favourable prognosis along with response to chemotherapy provides a potential to salvage bladder function in patients with locally advanced LELC. In a small number of patients with pure and predominant LELC treated with transurethral resection of bladder tumor (TUR) and chemotherapy only, no evidence of disease was achieved in a follow-up period between 2 to 18 years (8-10).

In most of the reported cases, the platinum-based chemotherapies were performed in the treatment of both locally advanced and metastatic disease (9). However, as with other variants of bladder tumors, there are no clear guidelines for the treatment of LELC. Exactly because of the respectively favourable prognosis efforts should be given in the prompt diagnosis of this rare tumor, especially when occurs in the pure type.

The differential diagnosis includes chronic cystitis, malignant lymphoma, and poorly differentiated carcinoma with a prominent stromal lymphocytic infiltrate (10). However, in our case lymphoplasmacytic infiltrate was not sufficiently dense as to obscure the epithelial component, while the presence of an epithelial malignancy was clearly apparent.
In conclusion, more experience in the management of this rare tumour is needed in order to extract safe conclusions on its prognosis and provide clear guideline for its treatment.

\section{References}

1. Wenig BM. Squamous cell carcinoma of the upper aerodigestive tract: precursors and problematic variants. Mod Pathol. 2002; 15:229-254.

2. Terada T. Epstein-Barr virus associated lymphoepithelial carcinoma of the esophagus. Int J Clin Exp Med. 2013; 6:219-26.

3. Mayer EK, Beckley I, Winkler MH. Lymphoepithelioma-like carcinoma of the urinary bladder-diagnostic and clinical implications. Nat Clin Pract Urol. 2007; 4:167-71.

4. Amin MB, Ro JY, Lee KM. et al. Lymphoepithelioma like carcinoma of the urinary bladder. Am J Surg Pathol. 1994; 18:466-473.

5. Samaratunga H, Delahunt B. Recently described and unusual variants of urothelial carcinoma of the urinary bladder. Pathology. 2012; 44:407-18.

6. Tamas EF, Nielsen ME, Schoenberg MP, Epstein JI. Lymphoepithelioma-like carcinoma of the urinary tract: a clinicopathological study of 30 pure and mixed cases. Mod Pathol. 2007; 20:828-834.

7. Singh NG, Mannan AR, Rifaat AA, Kahvic M. Lymphoepitheliomalike carcinoma of the urinary bladder: report of a rare case. Ann Saudi Med. 2009; 29:478-481.

8. Holmäng S, Borghede G, Johansson SL. Bladder carcinoma with lymphoepithelioma-like differentiation: a report of 9 cases. J Urol. 1998; 159:779-782.

9. Amin MB, Ro JY, Lee KM, Ordóñez NG, et al. Lymphoepithelioma-like carcinoma of the urinary bladder. Am J Surg Pathol. 1994; 18:466-473.

10. Lopez-Beltrán A, Luque RJ, Vicioso L, et al. Lymphoepitheliomalike carcinoma of the urinary bladder: a clinicopathologic study of 13 cases. Virchows Arch 2001; 438:552-557.

\section{Correspondence}

Konstantinos N. Stamatiou, MD (Corresponding Author)

stamatiouk@gmail.com

Urology Department, Tzaneio Hospital

Afendouli 1 Ave 18536 Pireas, Attica, Greece

Georgios Christopoulos, MD

Department of Internal Medicine, Tzaneio Hospital, Pireas, Greece

Aikaterini Tsavari, MD

Kallirhoe Koulia, MD

Kassiani Manoloudaki, MD

Thivi Vassilakaki, MD

Pathology Department, Tzaneio Hospital, Pireas, Greece 